\title{
STK35L1 regulates Plasmodium infection and expression of cell cycle genes during liver stage of malaria
}

\section{Phulwanti Sharma}

Central University of Rajasthan

Inderjeet Kalia

National Institute of Immunology

Vibha Kaushik

Central University of Rajasthan

\section{Daniela Brünnert}

University Hospital of Würzburg

\section{Afshana Quadiri}

National Institute of Immunology

Mohammad Kashif

Infectious Diseases Laboratory, National Institute of Immunology

Kirti Chahar

Central University of Rajasthan https://orcid.org/0000-0001-8080-1745

\section{Akhil Agarwal}

Central University of Rajasthan

\section{Agam Prasad Singh}

National Institute of Immunology

Pankaj Goyal ( $\sim$ pankaj_bio@curaj.ac.in )

Central University of Rajasthan

\section{Article}

Keywords: Plasmodium, malaria, STK35L1

Posted Date: December 29th, 2020

DOI: https://doi.org/10.21203/rs.3.rs-133230/v1

License: (1) (1) This work is licensed under a Creative Commons Attribution 4.0 International License.

Read Full License 


\section{Abstract}

Protein kinases of both the parasite and host are crucial in parasite invasion and survival and might be drug targets against drug-resistant malaria. STK35L1 was among the top five hits in kinome-wide screening, suggesting a role in malaria's liver stage. The function of STK35L1 in malaria is not known yet. We found that STK35L1 was highly upregulated during the infection of P. berghei in HepG2 cells and mice liver. Knockdown of STK35L1 remarkably suppressed the sporozoite infection in hepatocytes. STAT3 is upregulated and phosphorylated during $P$. berghei sporozoites infection. We found that STAT3 activation is required for both STK35L 1 and STAT3 upregulation. Furthermore, ten cell cycle genes were upregulated in the sporozoite-infected hepatocytes. Knockdown of STK35L 1 completely inhibited the upregulation of these genes. We identified STK35L 1 as a host kinase that plays an obligatory role in malaria's liver stage. It may be a potential drug target against drug-resistant malaria.

\section{Introduction}

Malaria caused by Plasmodium sp. infection is endemic and remains one of the predominant infectious diseases in South-East Asia and sub-Saharan African regions ${ }^{1}$. The asymptomatic liver stage of Plasmodium infection is obligatory in the parasite's lifecycle, and various host factors are critical for invasion and replication of the parasite in hepatocytes ${ }^{2}$. Protein kinases of both the parasite and the host are pivotal players in parasite invasion and survival strategies and might be drug targets against drugresistant malaria ${ }^{3}$. A high throughput siRNA-based screening of 727 kinases by Prudencio et al. identified five hepatocyte kinases, viz. MET, PKCZ, PRKWNK1, SGK2, and STK35 that might regulate the infection of Plasmodium berghei. Among these kinases, STK35 (also known as STK35L1and CLIK1) was previously shown to interact with CLP-36, a PDZ-LIM protein, and actin stress fibers suggesting its involvement in actin dynamics ${ }^{4}$.The reorganization of the host cell actin cytoskeleton is one of the crucial factors for Plasmodium infection, indicating the role of STK35L 1 in malaria ${ }^{5,6}$. However, the function of STK35L 1 is not yet established in Plasmodium infection during the liver stage of malaria.

STK35L 1 is a member of the NKF4 (New Kinase Family 4) Ser/Thr kinases family and is classified in the group "Other" of the human kinome ${ }^{7}$ and is mainly localized in the nucleus and nucleolus ${ }^{8}$. Previously, we showed that nuclear actin is a binding partner of STK35L 1 in endothelial cells signifying that STK35L 1 might have a role in gene transcription, cell migration, and chromatin remodeling ${ }^{9}$. Moreover, in endothelial cells, the silencing of the STK35L 1gene drastically impaired cell migration ${ }^{9}$. A study showed that importin-a2 enhanced the expression of the stk35/1gene, and constitutive overexpression of STK35L 1 activates caspase-independent cell death under oxidative stress conditions ${ }^{10}$.STK35 (STK35L 1) knockout mice showed defects in eye development and were infertile ${ }^{11}$. STK35L 1 transcript was altered in colorectal cancer and cardiomyopathy ${ }^{12,13}$. All these studies suggest that STK35L 1 has diverse roles in the physiology and pathophysiology of various diseases. A cell cycle-specific gene array analysis revealed that three genes (CDKN2A, DDX11, and GADD45A) were strongly downregulated in STK35L1gene knockdown-endothelial cells ${ }^{9}$. Notably, a study showed that CDKN2A expression was highly upregulated 
during acute malaria infection ${ }^{14}$. It suggests that STK35L 1 might be associated with CDKN2A expression in malaria.

A study showed that STAT3 plays a critical role in malaria pathogenesis as Plasmodium berghei (P. berghei) infection leads to the activation of STAT3 ${ }^{15}$. Recently, it was shown that STAT3 regulates the expression of STK35L 1 in osteosarcoma, suggesting a link of STK35L 1 with STAT3 and malaria ${ }^{16}$. The STATs are the transcription factors, and their family is composed of seven members; STAT1, STAT2, STAT3, STAT4, STAT5A, STAT5B, and STAT6 ${ }^{17}$. STAT activation is mediated via phosphorylation of Tyr705 by activation of AK/STAT pathway leading to homodimerization and induce gene transcription by binding to a specific DNA-response element in the promoter region of target genes. Activation of STAT3 plays a central role during viral infection in the liver ${ }^{18}$. In various cell types, STAT3 gets phosphorylated and upregulates the expressions of cell cycle genes, including Cyclin B1 and Cdc2 ${ }^{19,20 . ~ I n f e c t i o n ~ o f ~ m i c e ~}$ with Plasmodium yoelii strains (Py17XNL, Py17XL) activated STAT1 and STAT3 via phosphorylation, on the first day after parasite inoculation ${ }^{21}$. It is unknown whether activation of STAT3 regulates the expression of STK35L 1 in Plasmodium infection during the liver stage of malaria.

Based on the previous studies from other groups and the report from our lab, we hypothesized that STK35L 1 might be a crucial player in the pathogenesis of Plasmodium infection during the liver stage of malaria, and STAT3 might play an essential role in STK35L 1 regulation. In this study, we showed for the first time that STAT3 mediates the upregulation of STK35L 1 after infection of P. berghei in hepatocytes. STK35L 1 inhibition leads to the reduction of P. berghei sporozoite infection in hepatocytes. Additionally, various cell cycle genes were upregulated during P. berghei infection, and STK35L 1 regulates their expression.

\section{Results}

\section{STK35L1 is highly upregulated in vitro and in vivo during P. berghei infection}

To gain an insight into the role of STK35L 1 in governing susceptibility to parasite infection in the liver stage of malaria, a hepatocyte cell line HepG2 were infected with P. berghei ANKA sporozoites. The expression of STK35L 1 was measured by $q P C R$, and we found that it was significantly upregulated ( 5 fold; $p<0.01$ ) after sporozoite infection in HepG2 cells (Figure 1A). The upregulation of STK35L 1 was further confirmed at the protein level by Western blotting using an anti-STK35L 1 antibody. We found an increased expression ( 2 fold) of STK35L 1 protein in infected HepG2 cells (Figure 1B; black arrow).

We asked whether infection of $P$. berghei sporozoites in mice liver would also enhance the expression of Stk35/1.Indeed, we observed a significant increase ( 2 fold; $p<0.03)$ of Stk35/1 gene expression in mice liver infected with P. berghei sporozoites. (Figure 1C). The parasite load in the mice livers was also determined by the copy number of the P. berghei $18 S$ rRNA in the infected and uninfected liver. The data showed that all the mice were infected, but the infection (parasite load) was highly variable (Figure 1D). In 
a nutshell these data suggest that the STK35L 1 upregulation during P. berghei infection might play a crucial role in facilitating the parasite's infection and growth during the liver stage of malaria.

\section{Inhibition of STK35L1 impairs the infection of host cells by Plasmodium sporozoites.}

We asked whether STK35L 1 is essential for P.berghei sporozoite infection in hepatocytes. We knocked down the expression of STK35L 1 using three STK35L 1 siRNAs and then measured the parasite load to address this question. We procured commercial siRNAs that were designed from different regions of STK35L 1. We confirmed the knockdown efficiency of these siRNAs via qPCR and Western blotting. Two of the siRNAs (siRNA1 and siRNA2) showed the highest knockdown efficiency ( 82\% and 87\%, respectively) for STK35L 1 (Figure 2A). The knockdown of the STK35L1 protein was also confirmed by immunoblotting (Figure 2B). These siRNAs(siRNA1 and siRNA2) were chosen for further studies. To investigate the role of STK35L 1 in P.berghei infection, HepG2 cells were treated with siRNA1 and siRNA 2 for 72 hours and then infected with P.berghei sporozoites for 48 hours. The scrambled siRNA was used as a negative control. Notably, we found that the sporozoite infection (parasite load) was significantly reduced to $\sim 20 \%$ in cells treated with siRNA1 and siRNA 2 (Figure 2C). The infection was decreased slightly but was non-significant in cells treated with scrambled siRNA (Figure 2C).

Similarly, the STK35L 1 upregulation was significantly reduced after sporozoites infection in cells treated with siRNA1 and siRNA2 (Figure 2D). Scrambled siRNA could not affect the expression of stk35/1 (Figure 2D). These data showed the physiological relevance of STK35L 1 as a vital host factor required for the liver infection with Plasmodium sporozoites.

\section{STAT3 is highly upregulated during Plasmodium infection in HepG2 cells.}

We hypothesized that the STK35L1 upregulation during P. berghei infection is regulated by the member(s) of the STAT transcription factor family. First, we examined the expression of STAT family transcription factor genes in HepG2 cells via qPCR. We found that STAT1 and STAT3 genes were highly abundant in HepG2 cells (Figure 3A). The STAT4 gene was relatively less expressed ( 6 fold lower) in comparison to STAT1. Other family members (STAT2, STAT5A, STAT5B, and STAT6) were expressed at 50 to a 100-fold lesser extent in HepG2 cells (Figure 3A). We further studied the effect of sporozoite infection on STAT1, STAT3, and STAT4 expression in HepG2 cells. Notably, we found that only the expression of STAT3 was significantly upregulated ( 7 fold; $p<0.01$ ) in infected HepG2 cells (Figure $3 B$ ). We could not find any significant changes in the expression levels of STAT1 andSTAT4 (Figure 3B). The upregulation of STAT3 in infected cells was also confirmed by Western blotting using the anti-STAT3 antibody (Figure 3C). Collectively, these data show that only STAT3 is significantly upregulated via Plasmodium sporozoite infection, and STAT3-dependent signaling might be necessary for the liver stage of malaria.

\section{STAT3 is activated during Plasmodium infection in HepG2 cells and autoregulates its expression}

We investigated whether sporozoite infection activates STAT3 via phosphorylation in addition to enhancing its expression. We could observe that the phosphorylation of STAT3 residue tyrosine-705 was 
increased after sporozoite infection in HepG2 cells (Figure 4A). We found a very low STAT3 phosphorylation in non-infected cells (Figure 4A). These data indicate that sporozoite infection leads to STAT3 phosphorylation, which might regulate various host gene expressions.

Furthermore, to examine whether STAT3 activation regulates its expression, we used a specific STAT3 activator ML115 ${ }^{22}$. Notably, ML $115(4 \mu M)$ could significantly increase the expression of STAT3 by $\sim 3$ fold; $(p<0.002)$ within 6 hours of treating HepG2 cells with ML 115 (Figure 4B). To confirm that STAT3 regulates ML 115-dependent its upregulation, we used highly specific STAT3 inhibitors Stattic and 5,15$D P P^{23,24}$. We observed that both inhibitors could not significantly affect the basal expression of STAT3 (Figure 4B). As expected, both inhibitors successfully inhibit the ML 115-dependent upregulation of STAT3 by $\sim 85 \%$ and $\sim 80 \%$, respectively (Figure $4 B$ ). Together, these data suggest that activation of STAT3 via sporozoite infection leads to the upregulation of its expression in a positive feedback manner.

\section{Activation of STAT3 regulates the expression of STK35L1 in HepG2 cells.}

We hypothesized that STAT3 activation might regulate the STK35L 1 expression. We treated the HepG2 cells with specific STAT3 inhibitors, Stattic, and 5,15-DPP. Interestingly, the basal expression of STK35L 1 was significantly down-regulated after the treatment of Stattic $(\sim 60 \% ; p<0.013)$ and 5,15-DPP ( 85\%; $p<0.0025)$ (Figure 5A). This result suggests that STK35L 1 expression is tightly regulated via STAT3. Furthermore, we asked whether activation of STAT3 upregulates the expression of STK35L1. We

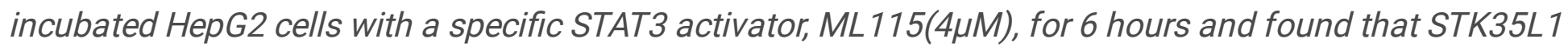
was significantly upregulated ( 2 fold; $p<0.005$ ) after the treatment of cells with ML 115 (Figure 5B). Next, we examined whether ML115-dependent upregulation of the STK35L 1 gene can be reverted after the inhibition of STAT3 activation. Indeed, Stattic, and 5,15-DPP could completely block the upregulation of STK35L 1 (Figure 5B). These data collectively confirm that STAT3 is the major transcription factor that plays a crucial role in regulating the expression of STK35L1. The STAT3/STK35L 1 axis might be critical for the liver stage of malaria.

\section{Various cell cycle genes were upregulated during Plasmodium infection, which were regulated via STK35L1}

Previously, we found that STK35L 1 regulates various cell cycle-related genes in endothelial cells ${ }^{9}$. To identify the role of STK35L 1 upregulation during P. berghei infection, we checked the expression of 11 genes (Table S2) during sporozoite infection. Interestingly, 10 genes (RAD51, MKI67, CDKN3, CDKN2A, CDK6, CDC20, DDX11, GADD45A, CCNB2, and CDC2) were significantly upregulated after sporozoite infection in HepG2 cells (Figure 6 and Table S2), but GTSE1 was not significantly upregulated (Figure 6; bottom right panel). Interestingly, RAD51 was upregulated 18 fold $(p<0.0001)$ in infected cells (Figure 6; top left panel). CDK6, DDX11, and CDC2 were 4-5fold upregulated after sporozoite infections (Figure 6). Moreover, GADD45A, CDC20, and CDKN2A were 3-4 fold upregulated (Figure 6). CDKN3, MKI67, and CCNB2 were $\sim 2-2.5$ fold upregulated (Figure 6). To summarize, these data indicate that these cell cyclerelated genes might play a crucial role in hepatocyte growth and, consequently, on malaria's liver stage. 
We asked whether STK35L 1 has a role in regulating the infection-dependent upregulation of these genes. Indeed, upregulation of the studied genes except CDC2 and GTSE1 was significantly downregulated after knockdown of the STK35L 1 gene (Figure 6). The upregulation of CDC2 after sporozoites infection is independent of STK35L1. These data signify that STK35L 1 is a major factor that controls the parasite infection-induced expressions of cell cycle-related genes.

\section{Discussion}

Protein kinases are emerging as new drug targets for therapeutic intervention against drug-resistant malaria. It is now evident that Plasmodium infection during the liver stage of malaria drastically changes the cell signaling within hepatocytes. In this study, we deciphered a new host cell signaling network that regulates the sporozoite infection during the liver stage of malaria. We identified a novel kinase, STK35L1, as one of the significant host kinases that play an obligatory role in malaria's liver stage.

The knowledge about the role of host kinases in the liver stage of malaria is mostly unknown. Only a few kinases were identified via kinome-wide high throughput screening, which might be crucial in the liver stage infection 25, 26. In a study, STK35 (STK35L1) was found among the top five hits during hepatocytes infection by $P$. berghei sporozoites ${ }^{26}$. The information about the functions of STK35L 1 in various diseases is minimal, and its role in malaria was utterly unknown. In this study, we confirmed that the knockdown of STK35L 1 remarkably suppressed the sporozoite infection in the hepatocyte cell line, HepG2. Interestingly, we found that STK35L 1 expression was enhanced in infected hepatocytes during liver stage infection in mice. These findings suggest a new and crucial role of STK35L 1 in the liver stage of malaria.

It is evident from previous studies that STAT3 transcriptionally regulates STK35L 1 expression in osteosarcoma cells ${ }^{16}$. Activation of STAT3 has been reported in the pathogenesis of severe malaria and viral infections, including human cytomegalovirus and hepatitis $B$ in hepatocytes ${ }^{15,27,28,29}$.In line with this, we proposed that the STAT3/STK35L 1 axis is active during the liver stage of malaria. First, we checked the basal expression of STAT family members in Hep 22 cells and found that STAT1, STAT3, and STAT4 were highly expressed compared to other family members. Notably, we showed the first time that STAT3 is autoregulated during P. berghei sporozoites infection. Additionally, we found that STAT3 is upregulated and phosphorylated at the residue, $Y-705$, which induces dimerization of STAT3 and then activates the expression of STAT3-targeted genes ${ }^{30}$. Together with previous studies, these data indicate that STAT3 signaling might play a pivotal role in the pathogenesis of the liver stage of malaria via controlling the STK35L 1 expression.

The small molecules that selectively inhibit or activate STAT3 can act as useful tools to study the STAT3specific signaling in host-pathogen interaction during malaria. To further extend our study, we used selective STAT3 inhibitors, Stattic,5,15-DPP, and ML115, a specific pharmacological activator of STAT3 signaling 22, 23,24. To support our findings, we mimicked the state of infection in hepatocytes by activating STAT3 signaling using ML115. Indeed, we found the upregulation of STK35L 1 and STAT3 in 
ML115-treated cells. Further, the upregulation was entirely blocked by Stattic and 5,15-DPP. These data confirm that STAT3 activation is crucial for STK35L 1 upregulation during sporozoite infection in hepatocytes.

It is not well understood whether Plasmodium sporozoites alter the cell cycle genes in hepatocytes for their benefit or it requires a specific stage of the host cell cycle for infection. A previous study showed that Plasmodium infection during the liver stage of malaria does not require host cell cycle progression ${ }^{31}$. Interestingly, transcriptional profiling of host cells during the liver stage infection at different time points revealed that various cell cycle linked genes were differentially expressed, suggesting cell cycle genes' role in survival and expansion of parasite growth and development ${ }^{32,33}$. Another study showed that Plasmodium introduced circumsporozoite protein in hepatocyte cytoplasm that regulates the expression of various genes including cell cycle-related genes to promote parasite growth ${ }^{34}$. Additionally, various studies indicate that STK35L 1 has a role in multiple cellular functions, including cell migration, apoptosis, and cell cycle 9, 10. Previously, we showed that STK35L 1 regulates 11 cell cycle genes in endothelial cells (Table S1) ${ }^{9}$.It indicates that the STAT3/STK35L 1 axis might regulate the cell cycle-related gene expression during sporozoite infection in hepatocytes.

We focused on the previously identified cell cycle genes controlled by STK35L $1^{9}$ and found that ten cell cycle genes were differentially expressed in the sporozoite-infected hepatocytes. Earlier, it was reported that cell cycle genes, GTSE1, CDC20, MKI67, CDC2, and CDKN3, were highly upregulated within 6 hours of infection in HepG2 cells ${ }^{33}$. We also found the upregulation of these genes except GTSE1 after 48 hours post-infection. A study identified that cell cycle regulatory genes (58 genes, including GADD45A) were differentially expressed after 18 hours of. infection ${ }^{32}$. Another report showed that CDKN2Awas highly expressed during acute malaria ${ }^{14}$. Besides these genes, we identified additional genes, viz. RAD51, DDX11, CDK6, and CCNB2 were highly upregulated in liver-stage parasite-infected hepatocytes. Notably, knockdown of STK35L 1 completely inhibited the upregulation of studied cell cycle genes except CDC2 gene. These data indicate that cell cycle genes facilitate the infection and growth of Plasmodium in hepatocytes, and the expression of these genes is regulated via STK35L 1.

This study established a new signaling axis STAT3/STK35L 1-dependent differential gene expression of specific cell cycle genes and the necessity of this axis for Plasmodium infection and proliferation during the liver stage of malaria.

Hepatocyte apoptosis is crucial for Plasmodium survival, and it must be regulated ${ }^{35,36}$. Interestingly, STK35L 1 holds caspase-independent cell death in stress conditions ${ }^{10}$. The role of STK35L 1 in hepatocyte cell death during sporozoite infection may be explored in future studies. Furthermore, STK35L 1 interacts with nuclear actin and actin stress fibers. Notably, the host cell actin cytoskeletal reorganization is critical for Plasmodium infection during the liver stage of malaria ${ }^{6}$. Nuclear actin plays a significant role in chromatin remolding, transcriptional regulation, and DNA repair ${ }^{37}$. The highly upregulated genes RAD51 and DDX11 38,39 are involved in DNA replication and DNA repair, and might 
play role in repair of host damaged DNA, arising due to pathogenic condition. Based on our findings, we propose a functional model of STK35L 1 role in malaria's liver stage parasite growth (Figure 7). This study will provide a new opportunity to develop STK35L 1 as a potential therapeutic drug target that could be used to treat drug-resistant malaria and improve clinical outcomes while reducing malaria mortality.

\section{Materials And Methods}

\section{Materials}

Primers were procured from Eurofins, India, ML115, a specific STAT3 activator, and STAT3 inhibitors, stattic and 5,15-DPP (5,15-Diphenylporphyrin), were procured from Cayman chemicals, USA. All cell culture reagents were purchased from HyClone, GE life sciences, USA. Scrambled siRNA and STK35L 1 gene-specific siRNAs were procured from Sigma-Aldrich, USA. STAT3, $p$-STAT3 (Y-705), and GAPDH antibodies were purchased from Santa Cruz Biotechnology, USA. Anti-mouse IRDye800 coupled secondary antibodies were purchased from LI-COR Biosciences, USA. Anti-STK35L 1 antibody (STK35-P1, rabbit) was established in our lab as described in supplementary method.

\section{Mosquito rearing and sporozoite production}

C57BL/6 mice were bred in institute facilities as per the guidelines of the Institutional Animal Ethics Committee (IAEC) of the National Institute of Immunology (NII), and all the experiments were carried out following the CPCSEA guidelines (Govt. of India). GFP expressing Plasmodium berghei ANKA (P. berghei ANKA) sporozoites strain was obtained from BEI Resources Repository, NIAID, NIH: MRA-671, contributed by M. F. Wiser. P. berghei ANKA parasites were cycled between mice and Anopheles stephensi mosquitoes. Mosquitoes (3-5 days old, female) were starved overnight and fed on six to eight-week-old C57BL/6 mice. After infection, mosquitoes were kept at $19^{\circ} \mathrm{C}, 70 \%$ to $80 \%$ relative humidity, and 12 hours light cycle for 18 days. The mosquitoes were fed on a $20 \%$ sucrose solution-soaked cotton pad during this period. After 18 days, mosquitos were washed with $50 \%$ ethanol and then with PBS (phosphatebuffered saline) three times. These washed mosquitoes were dissected in complete DMEM (Dulbecco's modified Eagle's medium) without antibiotics. Salivary glands were isolated and ground gently in complete DMEM to release sporozoites. Mosquito debris was removed by centrifuging at $100 \times \mathrm{g}$ for 4 min at $4^{\circ} \mathrm{C}$, and the number of sporozoites in the supernatant was determined using a hemocytometer.

\section{Cell culture and HepG2 cell infection}

All in vitro experiments were conducted with the HepG2 cell line, which was routinely maintained in DMEM containing $10 \%$ ( $\mathrm{V} / \mathrm{v}$ ) fetal bovine serum (FBS), 100 units $/ \mathrm{ml}$ penicillin, and $100 \mu \mathrm{g} / \mathrm{ml}$ streptomycin (Invitrogen). Cells were maintained in a humidified incubator with $5 \% \mathrm{CO}_{2}$ and at $37^{\circ} \mathrm{C}$. For infection, cells were grown $\left(1 \times 10^{6}\right.$ cells/well) in 6-well plates. After 24 hours, sporozoites $\left(1 \times 10^{5}\right.$ sporozoites/well) were added, and then cells were harvested 48 hours post-infection. Infected cells were sorted via FACS (BD FACS Aria). Control (non-infected) and GFP parasite positive cells were lysed using Trizol reagent, and total RNA was isolated as per manufacturers' protocol. 


\section{In vivo infectivity assay.}

To perform in vivo infection, $1 \times 10^{4} \mathrm{P}$. berghei ANKA sporozoites were injected intravenously into C57BL/6 mice. After 48 hours of sporozoite infection, livers were isolated from infected and non-infected mice and then homogenized in denaturing solution (4M guanidine thiocyanate, $25 \mathrm{mM}$ sodium citrate $\mathrm{pH} 7,0.5 \%$ sarcosyl, and $0.7 \% \beta$-mercaptoethanol in DEPC treated water). Total RNA was isolated, and the liver parasite load was estimated by employing quantitative real-time PCR (qPCR) using parasite-specific 18SrRNA primers ${ }^{40}$.

\section{Quantitative real-time PCR ( $q P C R$ )}

CDNA was synthesized with an iScript cDNA synthesis kit (BioRad, USA) as per manufacturers' protocol. Gene-specific primers (Table S1) were designed using NCBI primer blast tool, and qPCR was performed on Roche Light Cycler 96 or Mastercycler ${ }^{8}$ ep realplex (Eppendorf, Germany) as described previously $41,42$. Cycling program: $7 \mathrm{~min}$ at $95^{\circ} \mathrm{C}$ and then $40 \mathrm{cycles}$ with $10 \mathrm{~s}$ at $95^{\circ} \mathrm{C}$ and $20 \mathrm{~s}$ at $60^{\circ} \mathrm{C}$. The specificity of the amplicons was analyzed by thermal dissociation curve and agarose gel electrophoresis. Data were normalized against the housekeeping gene, $\beta$-actin.

\section{Gene silencing}

The HepG2 cells ( $5 \times 10^{4}$ cells/well) were grown in a 24-wellplate. STK35L 1 specific siRNA(50pmol) or scrambled siRNA (50pmol) were transfected in duplicates using Lipofectamine 2000 transfection reagent (Invitrogen) as described previously ${ }^{43}$. After 48-72 hours of transfection, cells were harvested, and knockdown efficiency was calculated at the gene level by qPCR and at the protein level using Western blotting.

\section{Western Blot}

Whole protein extracts were prepared in RIPA buffer (20mM HEPES; $p H$ 7.9,350mM NaCl, $1 \mathrm{mM}$ $\mathrm{MgCl}_{2}, 0.5 \mathrm{mM}$ EDTA,0.1mM, $1 \% \mathrm{NP}-40,0.5 \mathrm{mM} \mathrm{DTT,50mM} \mathrm{NaF,1} \mathrm{mM} \mathrm{Na}_{3} \mathrm{VO}_{4}, 0.2 \mathrm{mM} \mathrm{PMSF}$, and $1 \mu \mathrm{g} / \mathrm{mL}$ aprotinin) with protease and phosphatase inhibitors. Protein concentration was determined using a BCA protein kit (Thermo Scientific) as per the manufacturers' protocol. Equal amounts of protein were loaded in each well, resolved using SDS PAGE gel electrophoresis, and then transferred to nitrocellulose membranes (200voltsfor 60 min at $4^{\circ} \mathrm{C}$ ) using the Mini Trans-Blot electrophoresis unit (Bio-Rad). Membranes were blocked with Odyssey blocking buffer for an hour at room temperature and then probed at $4^{\circ} \mathrm{C}$ overnight with primary antibodies followed by secondary antibodies at room temperature for 1 hour. The dilution of primary antibodies was as follows: anti-STK35L 1(1:200) anti-STAT3(1:1000), anti-pSTAT3(Tyr-705; 1:1000) and anti-GAPDH (1:2000), anti-mouse IRDye800 coupled secondary antibodies (1:5000). The membrane was scanned using Odyssey Infrared Imager (LI-COR Biosciences), and densitometric analysis of the proteins was done using ImageJ (1.34s) software (NIH, Bethesda, MD). 


\section{Statistical analysis}

Each experiment was performed three or more times independently with different cell passages. Statistical analysis was performed on GraphPad PRISM 7 software (GraphPad, La Jolla, CA, USA) using one-way ANOVA or two-tailed unpaired t-test. The p-values of $<0.05$ were considered a statistically significant difference. Results are calculated as mean $\pm S D$, and data are shown as mean $+S D$.

\section{References}

1. World malaria report 2019. World Health Organization.

2. Raphemot R, Toro-Moreno M, Lu KY, Posfai D, Derbyshire ER. Discovery of Druggable Host Factors Critical to Plasmodium Liver-Stage Infection. Cell Chem Biol 26, 1253-1262 (2019).

3. Doerig C, et al. Malaria: targeting parasite and host cell kinomes. Biochim Biophys Acta Proteins Proteom 1804, 604-612 (2010).

4. Vallenius T, Makela TP. Clik1: a novel kinase targeted to actin stress fibers by the CLP-36 PDZ-LIM protein. J Cell Sci 115, 2067-2073 (2002).

5. Burda PC, Caldelari R, Heussler VT. Manipulation of the Host Cell Membrane during Plasmodium Liver Stage Egress. mBio 8, (2017).

6. Carrolo $\mathrm{M}$, et al. Hepatocyte growth factor and its receptor are required for malaria infection. Nat Med 9, 1363-1369 (2003).

7. Manning G, Whyte DB, Martinez R, Hunter T, Sudarsanam S. The protein kinase complement of the human genome. Science 298, 1912-1934 (2002).

8. Goyal P, Behring A, Kumar A, Siess W. Identifying and characterizing a novel protein kinase STK35L 1 and deciphering its orthologs and close-homologs in vertebrates. PLoS One 4, e6981 (2009). 
9. Goyal P, Behring A, Kumar A, Siess W. STK35L1 associates with nuclear actin and regulates cell cycle and migration of endothelial cells. PLoS One 6, e16249 (2011).

10. Yasuda $Y$, et al. Nuclear retention of importin alpha coordinates cell fate through changes in gene expression. EMBO J 31, 83-94 (2012).

11. Miyamoto $Y$, et al. The STK35 locus contributes to normal gametogenesis and encodes a IncRNA responsive to oxidative stress. Biol Open 7, 1-12 (2018).

12. Capra $M$, et al. Frequent alterations in the expression of serine/threonine kinases in human cancers. Cancer Res 66, 8147-8154 (2006).

13. Yang $X$, et al. Genome-wide linkage and regional association study of blood pressure response to the cold pressor test in Han Chinese: the genetic epidemiology network of salt sensitivity study. Circ Cardiovasc Interv 7, 521-528 (2014).

14. Asghar $M$, et al. Cellular aging dynamics after acute malaria infection: A 12-month longitudinal study. Aging Cell 17, (2018).

15. Liu M, et al. Heme mediated STAT3 activation in severe malaria. PLoS One 7, e34280 (2012).

16. Wu Z, Liu J, Hu S, Zhu Y, Li S. Serine/Threonine Kinase 35, a Target Gene of STAT3, Regulates the Proliferation and Apoptosis of Osteosarcoma Cells. Cell Physiol Biochem 45, 808-818 (2018).

17. Yu H, Jove R. The STATs of cancer-new molecular targets come of age. Nat Rev Cancer 4, 97-105 (2004). 
18. Roca Suarez AA, Van Renne N, Baumert TF, Lupberger J. Viral manipulation of STAT3: Evade, exploit, and injure. PLoS Pathog 14, e1006839 (2018).

19. Sun J, et al. E2F is required for STAT3-mediated upregulation of cyclin B1 and Cdc2 expressions and contributes to G2-M phase transition. Acta Biochim Biophys Sin (Shanghai) 51, 313-322 (2019).

20. Igelmann S, Neubauer HA. STAT3 and STAT5 Activation in Solid Cancers. Cancers 11, 1-19 (2019).

21. Shi X, Qin L, Liu G, Zhao S, Peng N, Chen X. Dynamic balance of pSTAT1 and pSTAT3 in C57BL/6 mice infected with lethal or nonlethal Plasmodium yoelii. Cell Mol Immunol 5, 341-348 (2008).

22. Madoux F, et al. Modulators of STAT Transcription Factors for the Targeted Therapy of Cancer (STAT3 Inhibitors). In: Probe Reports from the NIH Molecular Libraries Program). National Center for Biotechnology Information (US) (2010).

23. Schust J, Sperl B, Hollis A, Mayer TU, Berg T. Stattic: a small-molecule inhibitor of STAT3 activation and dimerization. Chem Biol 13, 1235-1242 (2006).

24. Uehara Y, Mochizuki M, Matsuno K, Haino T, Asai A. Novel high-throughput screening system for identifying STAT3-SH2 antagonists. Biochem Biophys Res Commun 380, 627-631 (2009).

25. Arang $N$, et al. Identifying host regulators and inhibitors of liver stage malaria infection using kinase activity profiles. Nat Commun 8, 1232 (2017).

26. Prudencio $M$, et al. Kinome-wide RNAi screen implicates at least 5 host hepatocyte kinases in Plasmodium sporozoite infection. PLoS Path 4, e1000201 (2008). 
27. Hu Z, Luo D, Wang D, Ma L, Zhao Y, Li L. IL-17 Activates the IL-6/STAT3 Signal Pathway in the Proliferation of Hepatitis $B$ Virus-Related Hepatocellular Carcinoma. Cell Physiol Biochem 43, 23792390 (2017).

28. Lepiller Q, Abbas W, Kumar A, Tripathy MK, Herbein G. HCMV activates the IL-6-JAK-STAT3 axis in HepG2 cells and primary human hepatocytes. PLoS One 8, e59591 (2013).

29. Liu M, Solomon W, Cespedes JC, Wilson NO, Ford B, Stiles JK. Neuregulin-1 attenuates experimental cerebral malaria (ECM) pathogenesis by regulating ErbB4/AKT/STAT3 signaling. J Neuroinflammation 15, 104 (2018).

30. Horvath CM. STAT proteins and transcriptional responses to extracellular signals. Trends Biochem Sci 25, 496-502 (2000).

31. Hanson KK, March S, Ng S, Bhatia SN, Mota MM. In vitro alterations do not reflect a requirement for host cell cycle progression during Plasmodium liver stage infection. Eukaryot Cell 14, 96-103 (2015).

32. Albuquerque SS, et al. Host cell transcriptional profiling during malaria liver stage infection reveals a coordinated and sequential set of biological events. BMC Genomics 10, 270 (2009).

33. Chattopadhyay R, et al. Early transcriptional responses of HepG2-A16 liver cells to infection by Plasmodium falciparum sporozoites. J Biol Chem 286, 26396-26405 (2011).

34. Singh AP, et al. Plasmodium circumsporozoite protein promotes the development of the liver stages of the parasite. Cell 131, 492-504 (2007).

35. Leiriao $P$, et al. HGF/MET signalling protects Plasmodium-infected host cells from apoptosis. Cell Microbiol 7, 603-609 (2005). 
36. van de Sand $C$, et al. The liver stage of Plasmodium berghei inhibits host cell apoptosis. Mol Microbiol 58, 731-742 (2005).

37. Caridi CP, Plessner M, Grosse R, Chiolo I. Nuclear actin filaments in DNA repair dynamics. Nat Cell Biol 21, 1068-1077 (2019).

38. Bonilla B, Hengel SR, Grundy MK, Bernstein KA. RAD51 Gene Family Structure and Function. Annu Rev Genet 54, 1-22 (2020).

39. Pisani FM, Napolitano E, Napolitano LMR, Onesti S. Molecular and Cellular Functions of the Warsaw Breakage Syndrome DNA Helicase DDX11. Genes (Basel) 9, 564 (2018).

40. Bruna-Romero O, Hafalla JC, Gonzalez-Aseguinolaza G, Sano G, Tsuji M, Zavala F. Detection of malaria liver-stages in mice infected through the bite of a single Anopheles mosquito using a highly sensitive real-time PCR. Int J Parasitol 31, 1499-1502 (2001).

41. Brünnert D, et al. Thrombin stimulates gene expression and secretion of IL-11 via protease-activated receptor-1 and regulates extravillous trophoblast cell migration. J Reprod Immunol 132, (2019).

42. Jaijyan DK, Singh H, Singh AP. A Sporozoite- and Liver Stage-expressed Tryptophan-rich Protein Plays an Auxiliary Role in Plasmodium Liver Stage Development and Is a Potential Vaccine Candidate. J Biol Chem 290, 19496-19511 (2015).

43. Goyal P, Brünnert D, Ehrhardt J, Bredow M, Piccenini S, Zygmunt M. Cytokine IL-6 secretion by trophoblasts regulated via sphingosine-1-phosphate receptor 2 involving Rho/Rho-kinase and Rac1 signaling pathways. Mol Hum Reprod 19, 528-538 (2013).

\section{Figures}


Figure 1

A

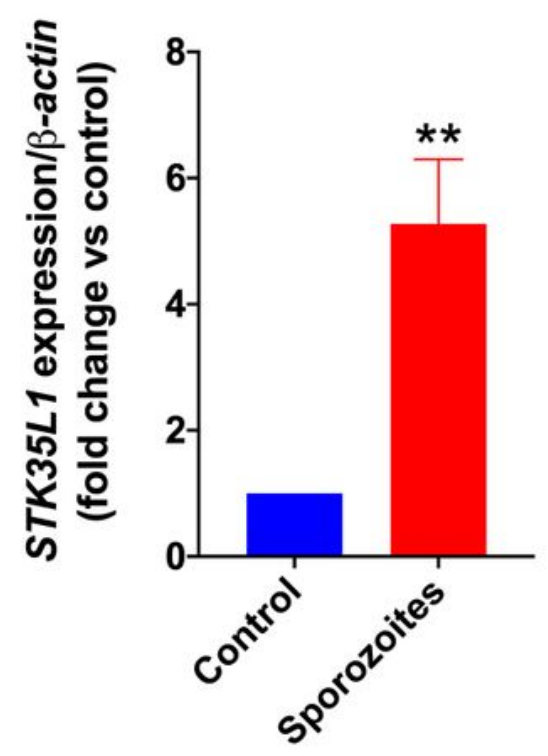

C

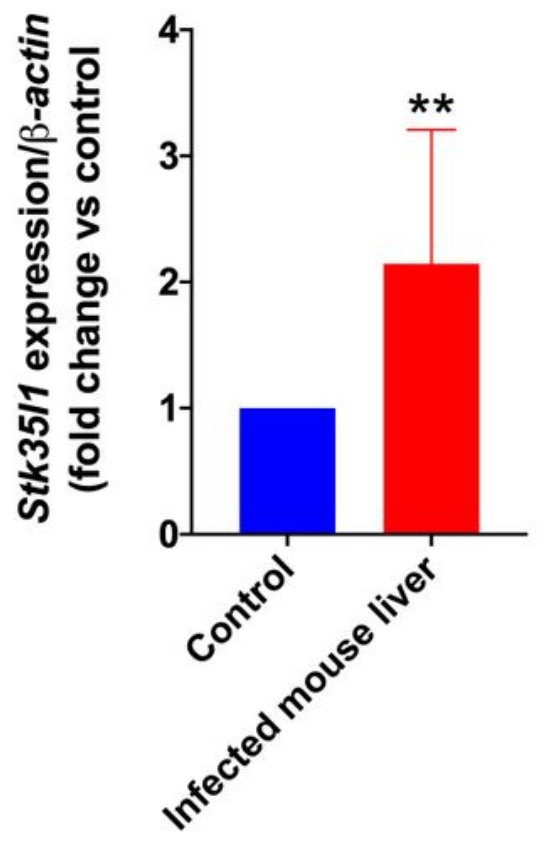

B

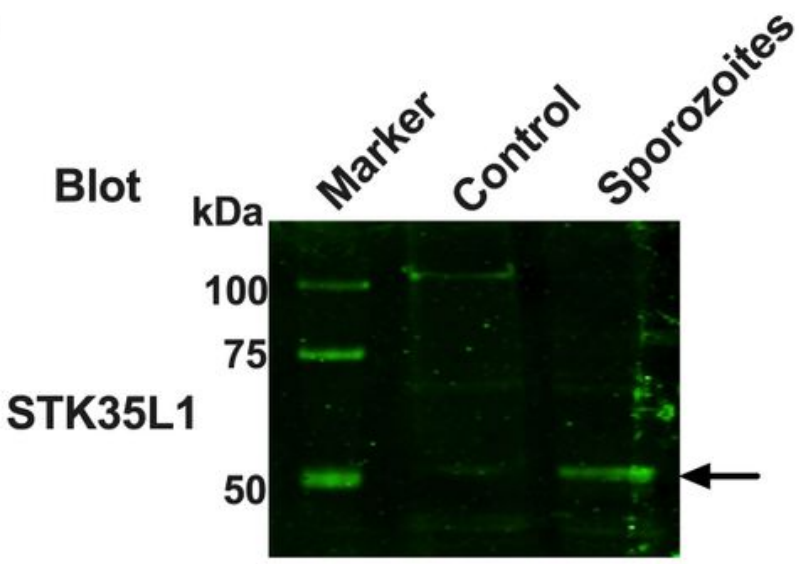

GAPDH $37 \longrightarrow$

D

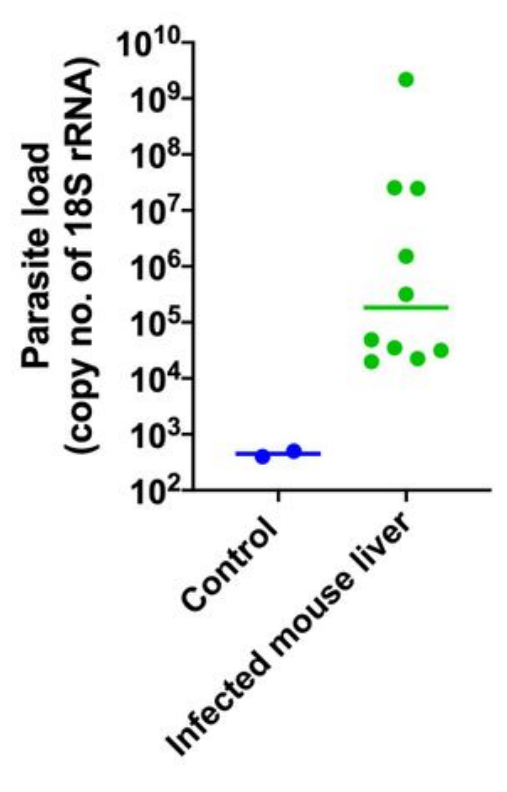

Figure 1

STK35L 1 is highly upregulated in vitro and in vivo during P. berghei infection. A) \& B) HepG2 cells were grown in a 6-well plate and infected with sporozoites (1x105 sporozoites/well). After 48 hours of infection, cells were harvested, and the expression of STK35L 1, at the gene and protein level, was measured by $q P C R$ and immunoblotting, respectively. Cells treated with media without sporozoites were used as a control. All data are expressed as the mean $\pm S D$ in 3 independent experiments. ${ }^{* *} p<0.01$. A) 
STK35L 1 was significantly upregulated in infected HepG2 cells ( $\$ 5$ folds; $p<0.01)$. $\beta$-actin was used as an internal control, and data were normalized against it. B) The whole cell lysate of infected and noninfected HepG2 cells were immunoblotted with anti-STK35L 1 antibody and anti-GAPDH antibody. GAPDH blot was used as a control for equal loading. These data show that the expression of STK35L 1 is highly upregulated after infection with sporozoites. C)\& D) C57BL/6 mice were infected with Plasmodium berghei ANKA sporozoites, and the total RNA was isolated from the liver after 48 hours of sporozoite infection. The expression of Stk35/1 was increased ( $\$ 2$ fold; $p<0.03)$ in infected mouse liver compared to the control mouse liver, as shown in the bar diagram. $\beta$-actin was used as an internal control. D) The sporozoite infection rate in the mouse liver was determined by qPCR using $18 S$ rRNA parasite primers. 
Figure 2

A

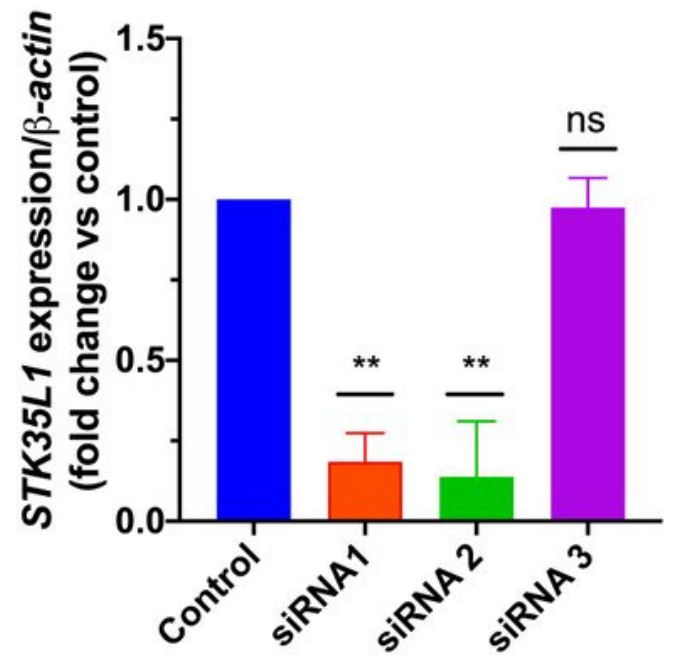

C

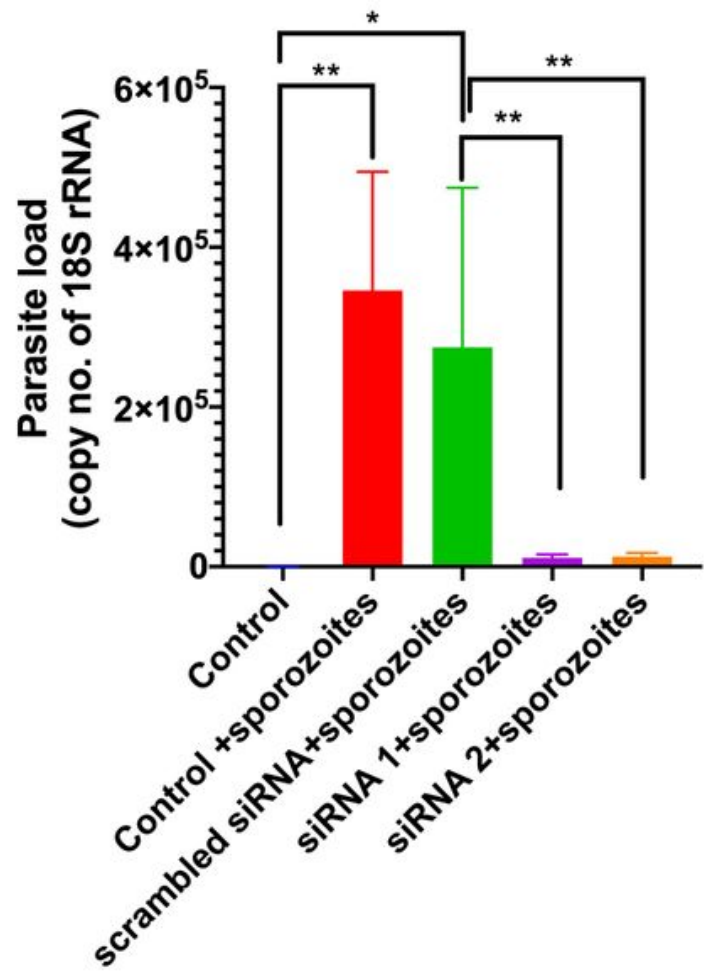

B

Blot

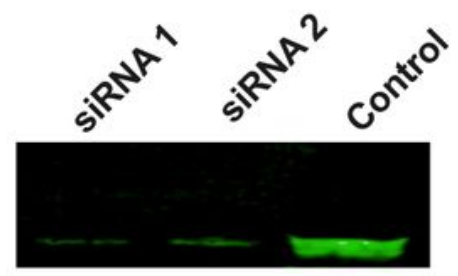

GAPDH
D

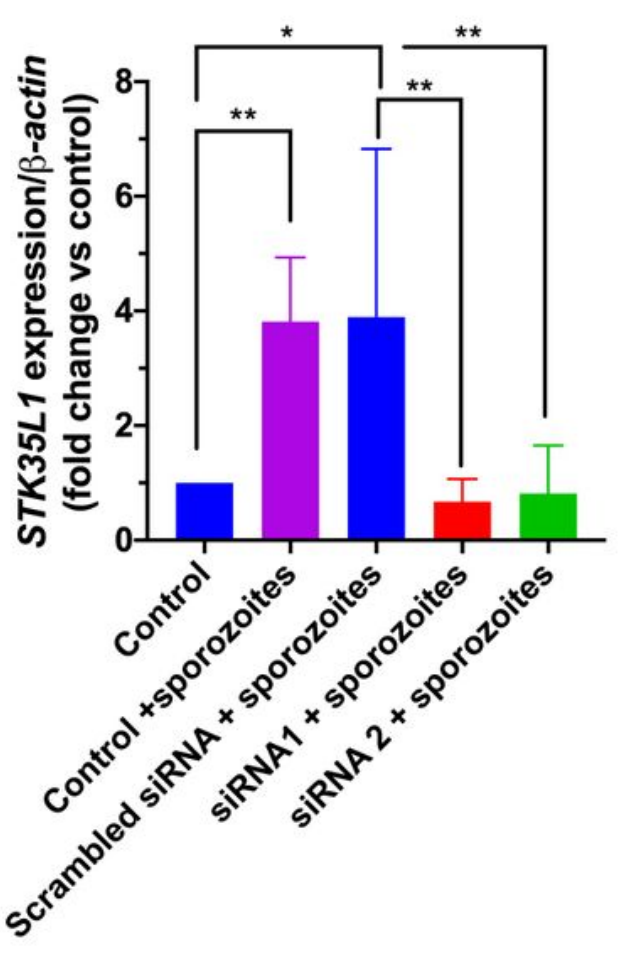

Figure 2

Inhibition of STK35L 1 impairs the infection of host cells by Plasmodium sporozoites. STK35L 1 was knocked down using specific siRNAs (siRNA1, siRNA2, and siRNA3), as described in the materials and methods. Cells transfected with scrambled siRNA were used as a control. Data were normalized against $\beta$-actin. All data are expressed as the mean $\pm S D$ in 3 independent experiments. ${ }^{*} p<0.05,{ }^{*} p<0.01$. A) Bar diagram shows the knockdown efficiency of the siRNAs. The siRNA1 and siRNA2 but not siRNA3 was 
able to significantly knockdown STK35L 1 (with an efficiency of $\sim 82 \%$ and $\sim 87 \%$, respectively). B) Immunoblot shows the expression of STK35L1 in control and siRNA1- and siRNA2-transfected HepG2 cells. The lower panel shows the immunoblot of GAPDH protein that was used as a control for equal loading. The siRNA1- or siRNA2-treated HepG2 cells and control cells were infected with P. berghei ANKA sporozoites, and the parasite infection was measured by qPCR after 48 hours of infection using $18 S$ rRNA parasite specific primers. Cells without any treatment served as controls. C) Bar diagram shows the parasite load in HepG2 cells. The knockdown of STK35L 1 with siRNA1 or siRNA drastically inhibited the parasite infection in HepG2 cells. D) Bar diagram shows the expression of STK35L 1 mRNA in HepG2 cells after knockdown of STK35L1, followed by sporozoite infection. The sporozoite infection in HepG2 cells could not enhance the expression of STK35L 1 in siRNA1-and siRNA2 -treated cells.

\section{Figure 3}

B

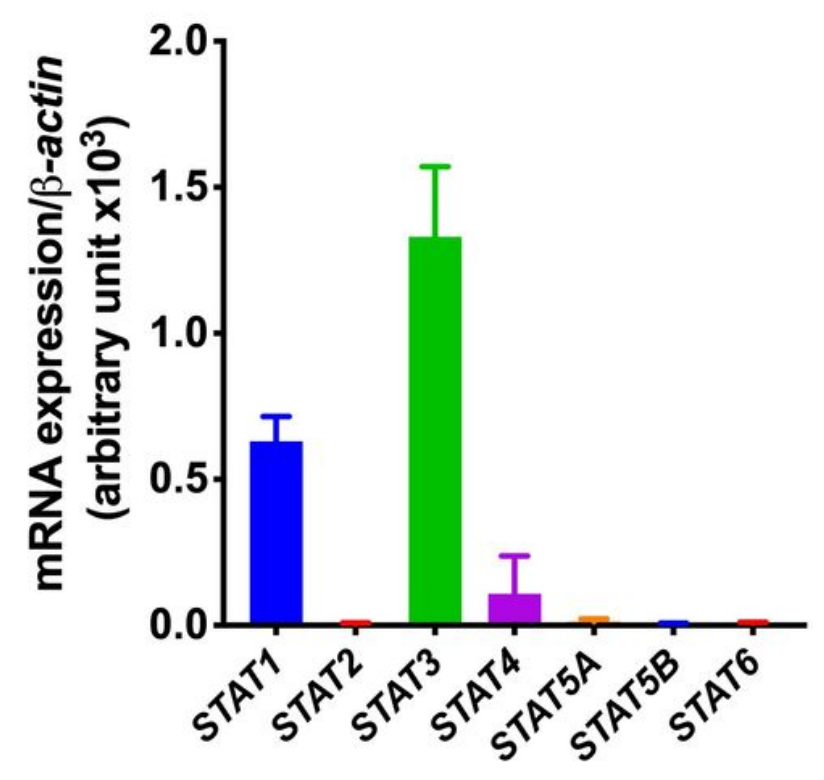

C

Blot
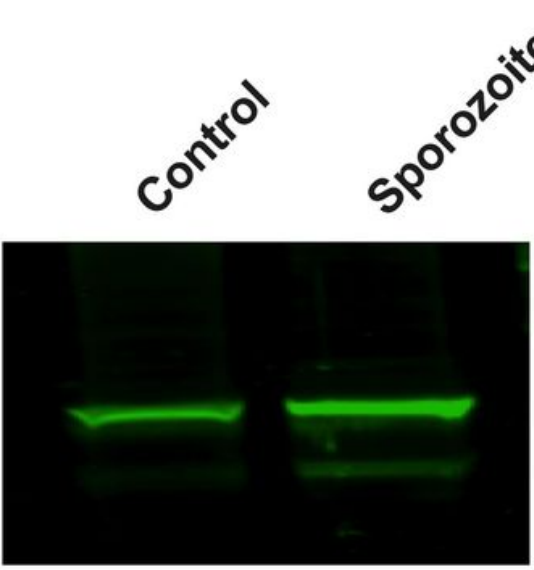

GAPDH

STAT3
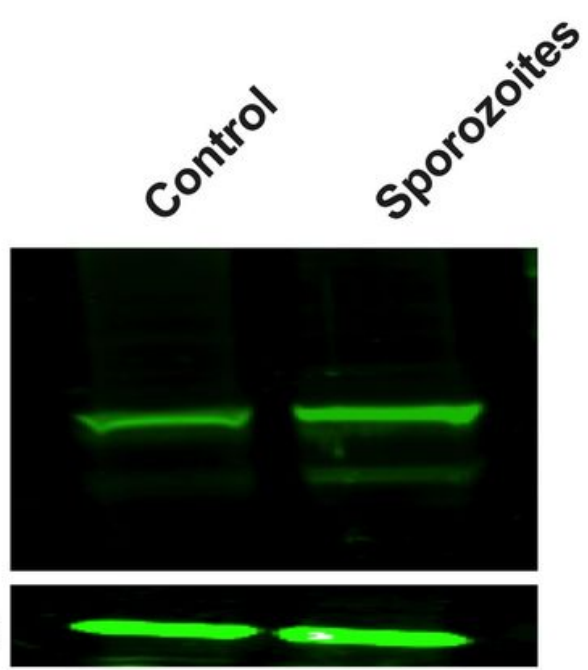

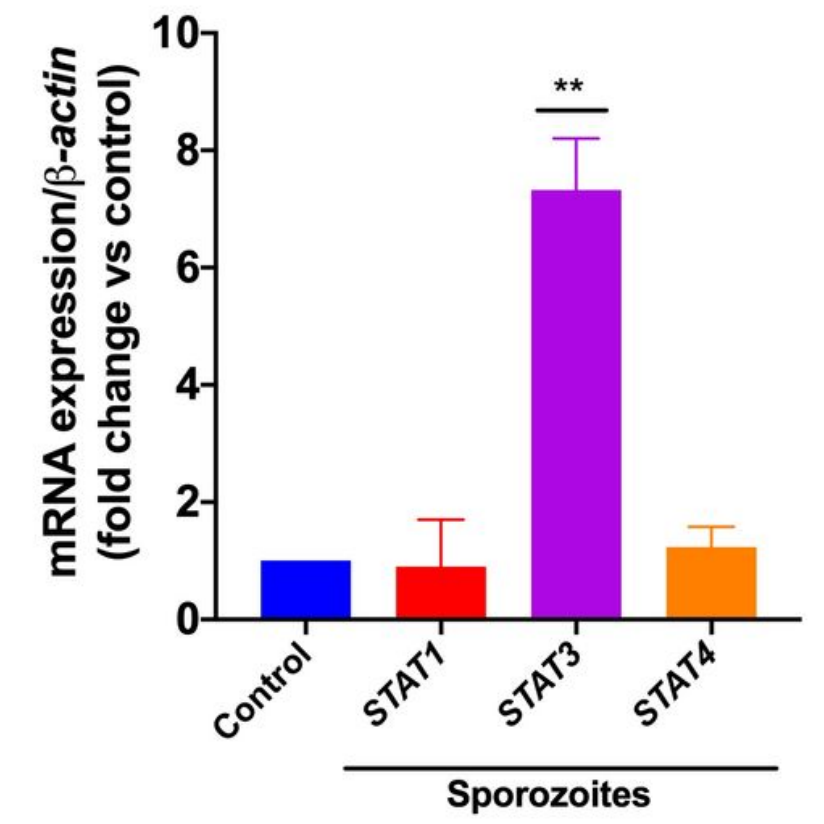

Sporozoites 


\section{Figure 3}

STAT3 is highly upregulated during Plasmodium infection in HepG2 cells. A) Bar diagram shows the basal expression of STAT family transcription factor genes (STAT1, STAT2, STAT3, STAT4, STAT5A, STAT5B, and STAT6) in HepG2 cells. These data show that STAT1, STAT3, and STAT4 genes are highly expressed. The other STAT family members, STAT2, STAT5A, STAT5B, and STAT6, were not detectable. HepG2 cells were infected with Plasmodium berghei ANKA sporozoites, and the expression of STAT1, STAT3, and STAT4 was measured using qPCR after 48 hours of infection. Data were normalized with the housekeeping gene, $\beta$-actin. ${ }^{*} p<0.01$. B) Bar diagram shows the expression of STAT1, STAT3, and STAT4 in infected and control HepG2 cells. The STAT3 was highly upregulated ( $\$ 7.3$ fold; $p<0.01)$ in sporozoite infected cells. C) Cell lysates of infected and non-infected HepG2 cells were immunoblotted with anti-STAT3 antibody and anti-GAPDH antibody. GAPDH blot was used as a control for equal loading. These data show that the expression of STK35L 1 is highly upregulated after infection with sporozoites.

\section{Figure 4}

A

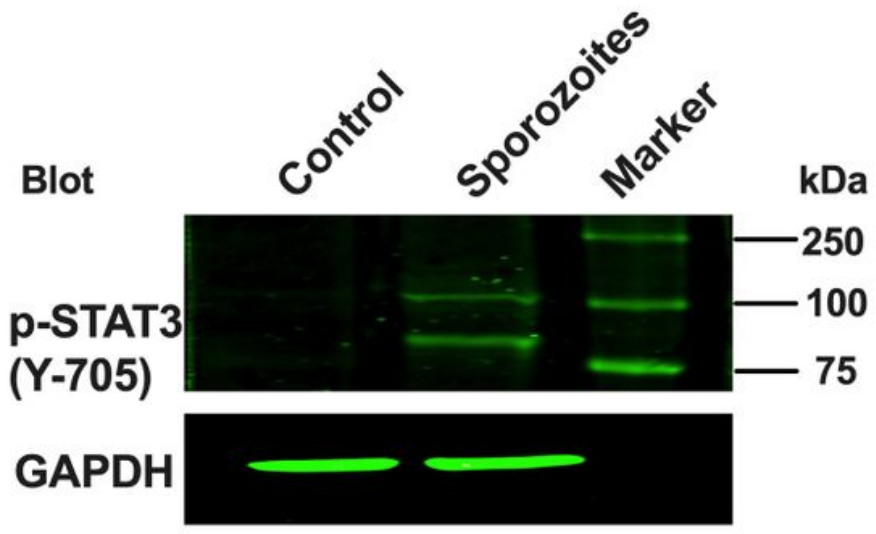

B

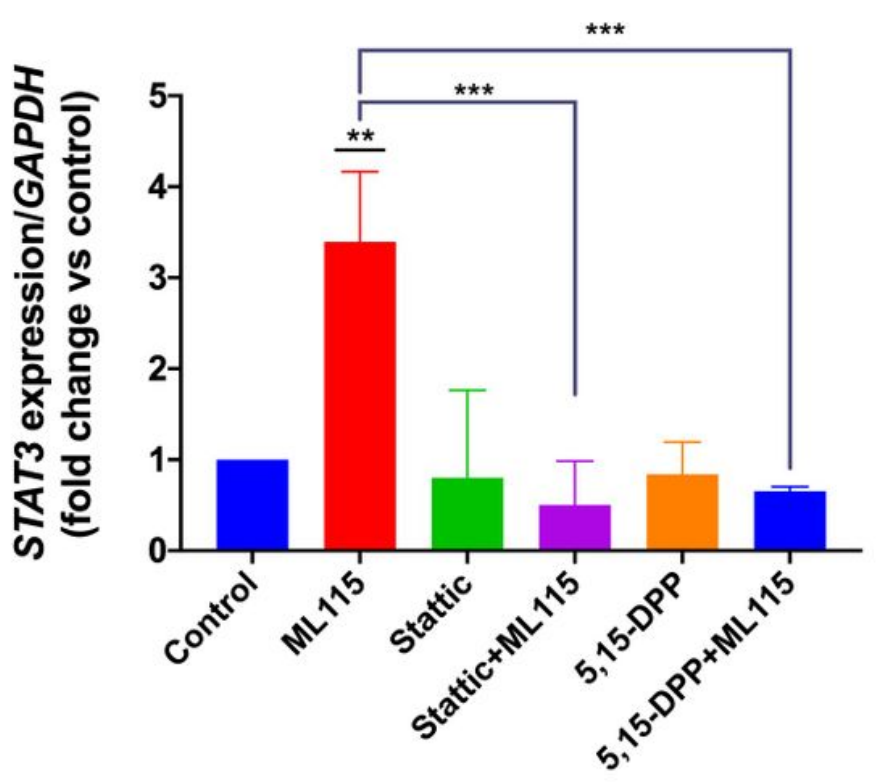

\section{Figure 4}

STAT3 is activated during Plasmodium infection in HepG2 cells and autoregulates its expression. HepG2 cells were infected with Plasmodium berghei ANKA sporozoites, and cells were harvested after 48 hours of infection. The expression of STAT3 was measured by qPCR, and STAT3 phosphorylation at residue, $Y$ 705, was detected by immunoblotting using a phospho-STAT3 antibody. A) A representative immunoblot shows the phosphorylation of STAT3 in infected and control cells. The data shows that the infection of sporozoites significantly enhanced the phosphorylation of STAT3. Immunoblot of GAPDH protein served as a control for equal loading. B) To confirm if the STAT3 expression is regulated via STAT3 activation, HepG2 cells were treated with a specific STAT3 activator, ML $115(4 \mu \mathrm{M})$, and the expression of STAT3 was 
measured by $q P C R$. Data were normalized against $\beta$-actin. ${ }^{* *} p<0.01,{ }^{* *} p<0.001$. The data shows that STAT3 expression was significantly increased ( $\$ 3$ fold; $p<0.002$ ) within 6 hours in ML 115-treated cells. ML115-dependent STAT3 upregulation is completely inhibited by highly specific STAT3 inhibitors, Stattic and 5,15-DPP.

\section{Figure 5}
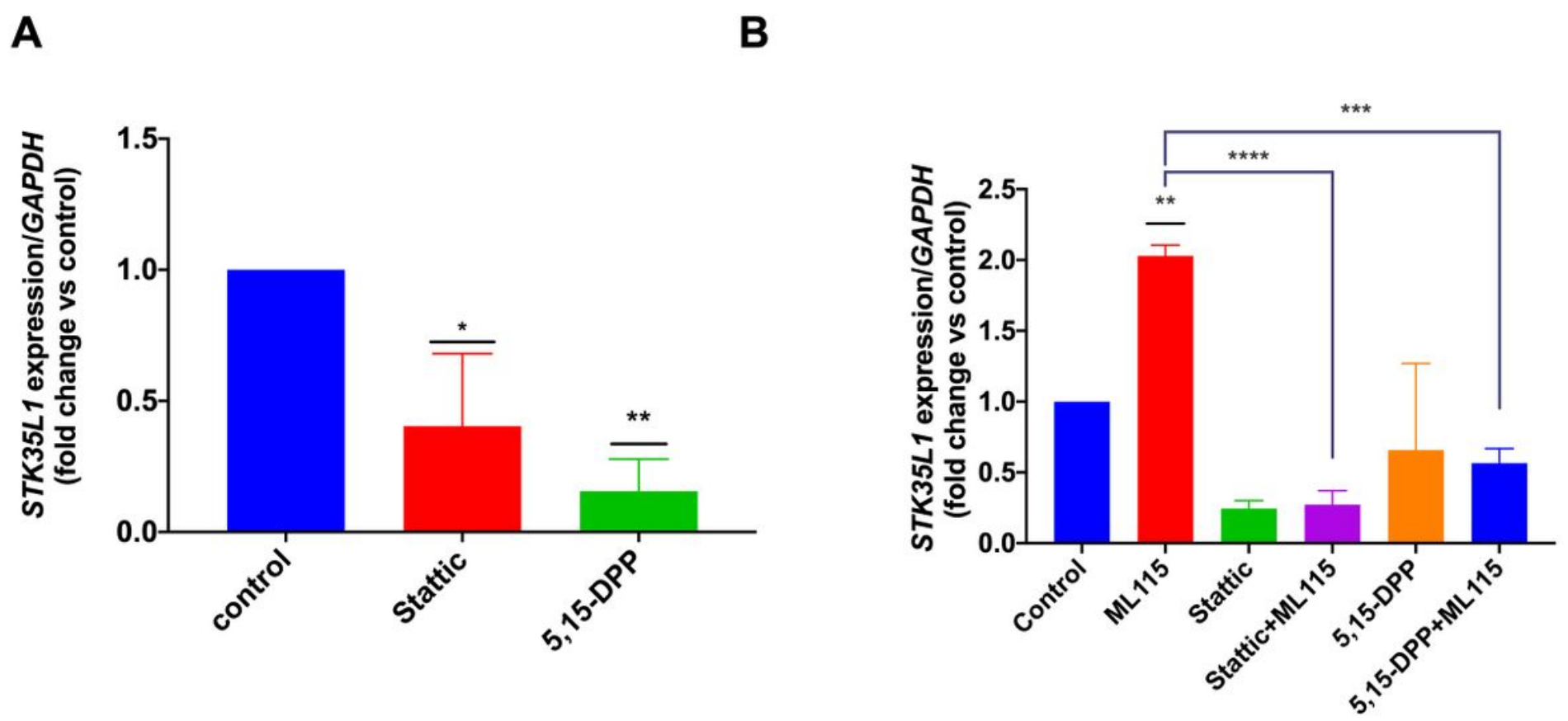

Figure 5

Activation of STAT3 regulates the expression of STK35L 1 in HepG2 cells. HepG2 cells were treated with pharmacological STAT3 inhibitors, Stattic $(10 \mu \mathrm{M})$ and 5,15-DPP (10 $\mu \mathrm{M})$, and a specific STAT3 activator, ML $115(4 \mu M)$, for 6 hours, and then STK35L 1 expression was measured by qPCR. Data were normalized against $\beta$-actin. ${ }^{*} p<0.05,{ }^{*} p<0.01,{ }^{* *} p<0.001$. A) The data show that the basal expression of

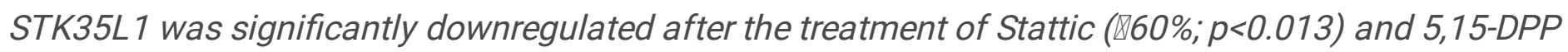
( $\$ 85 \% ; p<0.0025)$. B) Bar diagram shows that STK35L 1 was significantly upregulated ( $\$ 2$ fold; $p<0.005)$ after the treatment of cells with ML115. STAT3 inhibitors, Stattic and 5,15-DPP, could completely block the ML115-dependent upregulation of STK35L 1. 
Figure 6

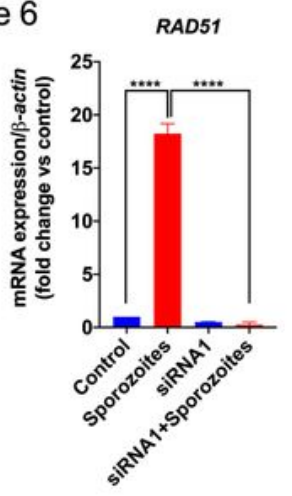

CDC2

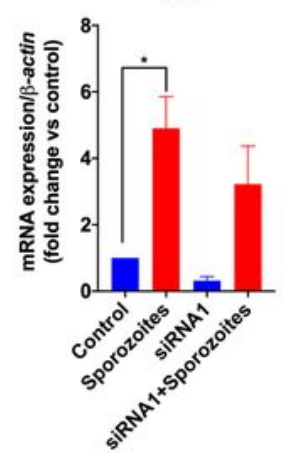

CDKN2A

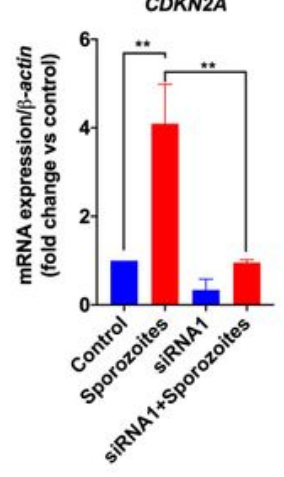

CCNB2

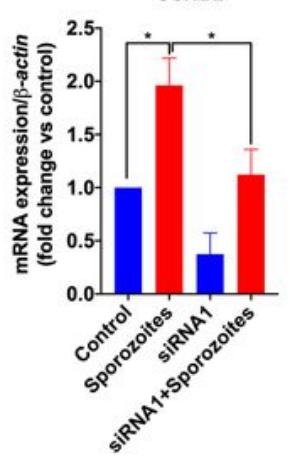

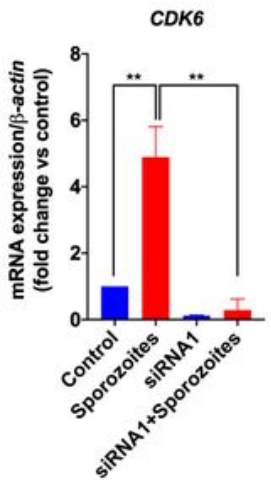

GADD45A

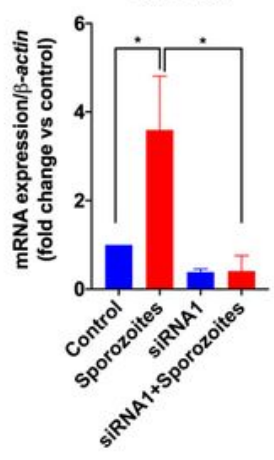

CDKN3

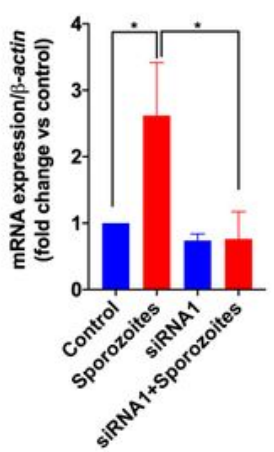

GTSE1

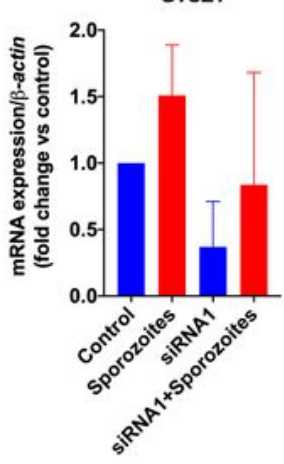

DDX11
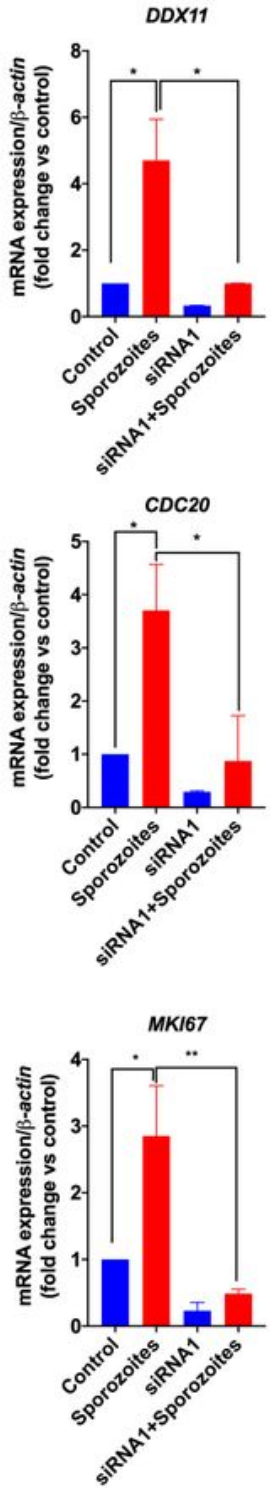

Figure 6

Various cell cycle genes were upregulated during Plasmodium infection in hepatocytes, which were regulated via STK35L1 HepG2 cells treated with siRNA1 for 72 hours, and the control cells were infected with P. berghei ANKA sporozoites and the expression of various cell cycle genes (Table S2) was measured by $q P C R$ after 48 hours of infection using $18 S$ rRNA primers. Cells without any treatment served as controls. Out of the 11 genes under study, 10 genes (RAD51, MKI67, CDKN3, CDKN2A, CDK6, CDC20, 
DDX11, GADD45A, CCNB2and CDC2) were significantly upregulated after sporozoite infection in HepG2 cells, while the upregulation of GTSE1 was not significant. The upregulation of all the genes in sporozoite-infected cells except CDC2 was downregulated considerably after knockdown of STK35L 1 using siRNA1.

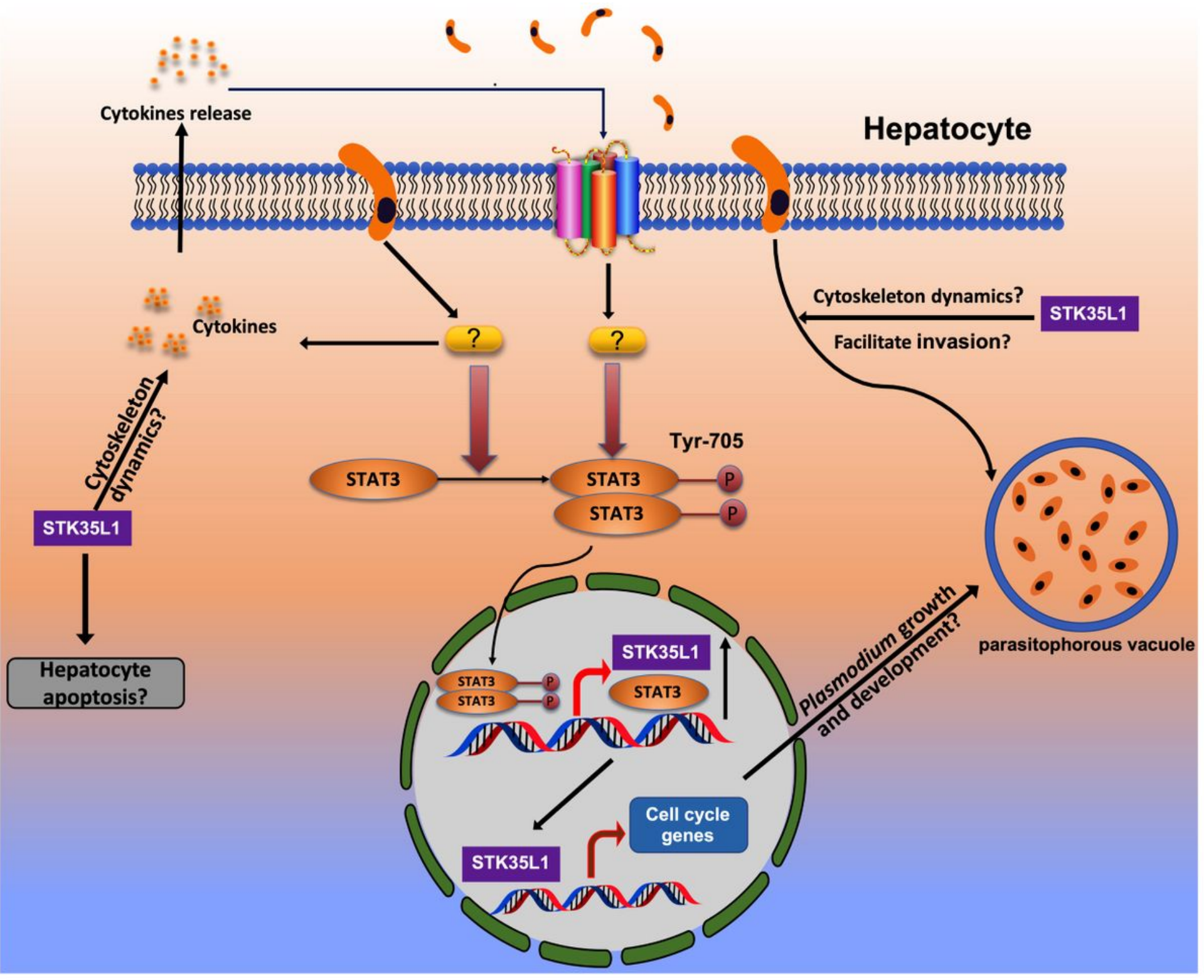

Figure 7

Proposed model of STK35L 1 functions during the liver stage of malaria. The model shows the potential role of STK35L 1 in the liver stage of malaria. The data from this study and the literature suggest that STK35L 1 might regulate gene transcription, apoptosis, and actin dynamics to facilitate the invasion of Plasmodium sporozoites and their growth in hepatocytes during the liver stage of malaria.

\section{Supplementary Files}


This is a list of supplementary files associated with this preprint. Click to download.

- MethodS1.docx

- Tables1andS2.docx 